\title{
A COMPARATIVE STUDY OF THE DIRECT BOUNDARY ELEMENT METHOD AND THE DUAL RECIPROCITY BOUNDARY ELEMENT METHOD IN SOLVING THE HELMHOLTZ EQUATION
}

\author{
SONG-PING ZHU ${ }^{\oplus 1}$ and YINGLONG ZHANG ${ }^{2}$
}

(Received 11 June 2006)

\begin{abstract}
In this paper, we compare the direct boundary element method (BEM) and the dual reciprocity boundary element method (DRBEM) for solving the direct interior Helmholtz problem, in terms of their numerical accuracy and efficiency, as well as their applicability and reliability in the frequency domain. For BEM formulation, there are two possible choices for fundamental solutions, which can lead to quite different conclusions in terms of their reliability in the frequency domain. For DRBEM formulation, it is shown that although the DBREM can correctly predict eigenfrequencies even for higher modes, it fails to yield a reasonably accurate numerical solution for the problem when the frequency is higher than the first eigenfrequency.
\end{abstract}

2000 Mathematics subject classification: primary 65N38; secondary 35Q35.

Keywords and phrases: the dual reciprocity boundary element method (DRBEM), Helmholtz equation, irregular frequencies.

\section{Introduction}

Many boundary value problems, such as those of wave propagation in solids and fluids, structure (membrane) vibrations, and those in acoustics, are represented by the Helmholtz equation

$$
\nabla^{2} \phi+k^{2} \phi=0
$$

\footnotetext{
${ }^{1}$ School of Mathematics and Applied Statistics, University of Wollongong, Wollongong NSW 2522, Australia; e-mail: spz@uow.edu.au.

${ }^{2}$ Department of Environmental \& Biomolecular Systems, OGI School of Science \& Engineering, Oregon Health \& Science University, Beaverton, OR 97006, USA

(C) Australian Mathematical Society 2007, Serial-fee code 1446-1811/07
} 
where $k$ is a positive constant (known as the wavenumber in wave propagation problems), and $\phi$ is an unknown function of two- or three-dimensional spatial coordinates. The problems with the Helmholtz equation as the governing equation can be divided into three groups; direct, eigenvalue and inverse problems. A direct problem involves the determination of the unknown function $\phi$ for a given boundary and the corresponding boundary conditions [22], whereas an eigenvalue problem involves the determination of the natural frequencies (eigenfrequencies) of the system [7]. On the other hand, in an inverse problem some kinds of inhomogeneities in the medium (for example, for given boundary conditions, find the shape of the inhomogeneous medium, or for a given shape of the inhomogeneous medium, find the boundary conditions, etc.) are to be determined under some given far-field conditions [5]. In most cases, as irregular boundaries are involved, numerical solutions are sought. Up to now, many numerical techniques have been proposed and developed for this type of equation, and these techniques can be categorized into three groups: the finite difference method [17], the finite element method [21,23], and the boundary element method (BEM) [9]. Although there are problems associated with boundary integral formulations of the problem (for example, irregular frequencies which will be discussed briefly later on), the BEM is still highly regarded as the most efficient method to deal with the Helmholtz equation, especially when an infinite domain is involved in the calculation [2].

Within the frame of the BEM, there are different formulations of boundary integrals. Due to their direct connection to physical quantities, direct formulations are usually more preferable to indirect formulations. There are also some variants of the BEM proposed to solve the Helmholtz equation more efficiently. For example, Nowak and Brebbia [13] proposed solving the Helmholtz equation with the Multiple Reciprocity Method (MRM) technique. Such an application, however, is efficient only if the nondimensionalized wavenumber is less than unity, which is usually much smaller than the first eigenfrequency (say, $\pi$ for the numerical example shown in their paper). Partridge and Brebbia [15] suggested that the Dual Reciprocity Boundary Element Method (DRBEM) be taken as another alternative for solving the Helmholtz equation. Although they have shown that the eigenvalues of interior Helmholtz problem can be obtained very accurately with the DRBEM even for higher modes, they did not mention the applicability of the method for solving a direct problem. In fact, not many studies have been conducted since then to show the accuracy of the DRBEM as well as the range of applicability of the method in the frequency domain for solving a direct Helmholtz problem; the accuracy of the DRBEM and its range of applicability in the frequency domain, particularly for large wavenumbers, remain to be examined.

The aim of this paper is to present a comparison of the BEM and the DRBEM in solving the Helmholtz equation (the direct problem or the eigenvalue problem) from the following four aspects: their accuracy and efficiency, their range of applicability 
in the frequency domain and their reliability in terms of avoiding the well-known "irregular frequencies". Since there are two possible choices of fundamental solutions for a direct BEM formulation of interior Helmholtz problems, that is, with either a Hankel function or a Neumann function (which is the real part of a Hankel function) as the weighting function [12], a comparison of the numerical results obtained from these two different fundamental solutions is also presented in this paper.

The paper is divided into four sections. In Section 2, the formulations of boundary integral equations adopting the BEM and the DRBEM respectively are briefly outlined, with the differences between them being highlighted. Then two numerical examples are presented in Section 3, to compare the BEM and DRBEM formulations in solving the Helmholtz equation. For the BEM formulation, two possible choices of fundamental solutions were also examined. Our conclusions are drawn in Section 4.

\section{Boundary Integral Formulation for the BEM and the DRBEM}

Suppose Equation (1.1) holds in a closed two-dimensional domain. A boundary value problem is constituted with the governing equation (1.1) and some properly prescribed non-complex boundary conditions on the boundary $\Gamma$, which is assumed to be a regular surface of Kellog type [8].

If the BEM is adopted, the corresponding boundary integral equation (BIE) (direct formulation) reads

$$
c_{\xi} \phi_{\xi}+\int_{\Gamma}\left(\frac{\partial \phi^{*}(p ; \xi)}{\partial n} \phi(p)-\phi^{*}(p ; \xi) \frac{\partial \phi(p)}{\partial n} \phi(p)\right) d \Gamma(p)
$$

where $c_{\xi}$ is a geometric parameter [3] associated with the location of the source point $\xi$, $\boldsymbol{n}$ is the outwards normal unit vector, $\boldsymbol{p}$ is a spatial point and $\phi^{*}$ is a fundamental solution of the Helmholtz equation. For two-dimensional problems, $\phi^{*}$ can be taken as either [12]

$$
\phi^{*}(\boldsymbol{p} ; \xi)=\frac{i}{4} H_{0}^{(1)}(k r(\boldsymbol{p} ; \xi))
$$

or

$$
\phi^{*}(\boldsymbol{p} ; \xi)=-\frac{1}{4} Y_{0}(k r(p ; \xi)),
$$

where $H_{0}^{(1)}$ is the Hankel function of the first kind of zero order, $Y_{0}$ is the Bessel function of the second kind (the Neumann function) of zero order, $i=\sqrt{-1}$ and $r(p ; \xi)$ is the Euclidian distance between points $p$ and $\xi$. The justification of the Neumann function (2.3) as the fundamental solution is obvious if one notices that (2.2) is a fundamental solution and its imaginary part (involving a Bessel function of the first kind) possesses 
no singularity at the source point. Furthermore, for an interior problem governed by the Helmholtz equation, no boundary conditions at infinity (usually some kind of radiation conditions) have to be satisfied by the fundamental solution. It should be noted that for exterior Helmholtz problems, (2.3) ceases to be a fundamental solution because it doesn't exhibit the appropriate behaviour at large distances and therefore it doesn't satisfy the radiation condition at infinity $[19,20]$. Clearly, the wavenumber $k$ is involved in both of the above two fundamental solutions $\phi^{*}$.

After the boundary is discretized into "elements" and within each element some polynomial shape functions are adopted to approximate the variation of $\phi$ and $\partial \phi / \partial n$, the boundary integrals in Equation (2.1) can be discretized so that the nodal values of $\phi$ and $\partial \phi / \partial n \equiv q$ are taken out of the integral signs.

Consequently, a linear algebraic system

$$
H \phi-G q=0
$$

is formed upon placing the source point $\xi$ at each boundary node (the collocation method) and carrying out the integral within each boundary element. In (2.4), the matrices $\boldsymbol{H}$ and $\boldsymbol{G}$ are of the conventional sense [3], and the vectors $\phi$ and $\boldsymbol{q}$ contain the values of $\phi$ or $q$ at each boundary node. It should be noted that if the complex fundamental solution (that is, the Hankel function) is adopted, then the matrices $\boldsymbol{H}$ and $\boldsymbol{G}$ are all complex. Furthermore, when the Hankel function is used as the weighting function, the elements of $\phi$ and $q$ must be assumed to be complex as well (the final solution is taken to be the real part of the corresponding complex variable). Otherwise this approach would be identical to the approach adopting the Neumann function as the fundamental solution. Finally, the unknowns contained in Equation (2.4) can be solved once the appropriate boundary conditions are imposed.

The formulation of the BIE for the Helmholtz equation with the adoption of the DRBEM, on the other hand, has been given by Partridge and Brebbia [15]. For ease of reference, it is again briefly outlined here. Firstly, Equation (1.1) is rewritten in the following form:

$$
\nabla^{2} \phi=-k^{2} \phi
$$

in which the Laplacian operator is now taken as the main differential operator, whose fundamental solution in the two-dimensional case is

$$
u^{*}(p, \xi)=-\frac{1}{2 \pi} \ln r(p, \xi) .
$$

Notice that now $k$ is no longer involved in the fundamental solution. Adopting $u^{*}$ as the weighting function, the weighted residual statement for Equation (2.5) reads

$$
\int_{\Omega} u^{*} \nabla^{2} \phi d \Omega=-k^{2} \int_{\Omega} u^{*} \phi d \Omega
$$


The idea of the DRBEM is to use a series of shape functions (or interpolation functions) to approximate the nonhomogeneous part of the governing equation (in our case, the right-hand side of Equation (2.5)) and thus to transform the domain integral into boundary integrals. Following Partridge and Brebbia [15], we let

$$
\phi=\sum_{j=1}^{N+L} f_{j} \alpha_{j}
$$

where $\alpha_{j}$ are some unknown coefficients to be determined by the collocation method, and $f_{j}$ are a series of interpolation functions with point $j$ located at one of $N$ boundary or $L$ internal collocation points. Here the simplest form for the interpolation function is adopted [15], that is,

$$
f_{j}=1+r_{j}
$$

in which $r_{j}$ is the distance from point $j$ to a field point. After placing the field point at each of $N$ boundary collocation nodes and $L$ internal collocation points, Equation (2.7) can be written in matrix form as

$$
\phi=F \alpha,
$$

from which the vector $\alpha$ can be determined.

Now, upon substituting Equation (2.7) back into the right-hand side of Equation (2.6) and making use of the property

$$
f_{j}=\nabla^{2} \hat{u}_{j},
$$

where $\hat{u}_{j}=r_{j}^{2} / 4+r_{j}^{3} / 9$, dual reciprocity is achieved in the sense that the domain integrals in Equation (2.6) can be transferred into boundary integrals only via Green's formula and a BIE is thus obtained in the form

$c_{\xi} \phi_{\xi}+\int_{\Gamma}\left(\phi q^{*}-u^{*} q\right) d \Gamma=-k^{2} \sum_{j=1}^{N+L}\left\{\alpha_{j}\left(c_{\xi} \hat{u}_{j \xi}+\int_{\Gamma}\left(\hat{u}_{j} q^{*}-u^{*} \hat{q}_{j}\right) d \Gamma\right)\right\}$,

in which $\hat{u}_{j \xi}$ is the value of $\hat{u}_{j}$ at point $\xi$ and $q^{*}$ and $\hat{q}_{j}$ are the normal derivatives of $u^{*}$ and $\hat{u}_{j}$, respectively. The rest of the steps are similar to those in BEM formulation, that is, after discretizing the boundary and placing the source point $\xi$ at each of $(N+L)$ collocation points, Equation (2.9) is transformed into a linear algebraic system, which can be written in matrix form as

$$
H^{D} \phi-G^{D} q=-k^{2}\left(H^{D} \hat{U}-G^{D} \hat{Q}\right) F^{-1} \phi
$$

where $H^{D}$ and $G^{D}$ are associated with the fundamental solution of Laplace's equation [3], and each column of $\hat{U}$ or $\hat{\boldsymbol{Q}}$ contains a vector $\hat{\boldsymbol{u}}_{j}$ or $\hat{\boldsymbol{q}}_{j}$, respectively. After 
utilizing boundary conditions, the unknown values $\phi$ (including those at $L$ internal points) and $q$ can be found from Equation (2.10).

An important feature of Equation (2.10) is that the matrices $H^{D}, G^{D}, \hat{U}, \hat{Q}$ and $F$ are all independent of the wavenumber $k$ and thus can be computed once and stored in a data file. Obviously this is extremely convenient for eigenvalue analyses, where $k$ needs to be changed from time to time. Furthermore, now that the fundamental solution is in terms of logarithmic functions instead of Bessel functions, the calculation of the matrices $\boldsymbol{H}^{D}$ and $\boldsymbol{G}^{D}$ in Equation (2.10) is much faster than that of $\boldsymbol{H}$ and $\boldsymbol{G}$ in Equation (2.4). This is the reason why the DRBEM is usually computationally more efficient than the BEM, even if a larger number of nodes (including boundary nodes and internal points) are used. In the next section, we shall compare, making use of the results of two numerical experiments, the numerical accuracy and efficiency of the BEM and the DRBEM in solving Helmholtz equations.

\section{Numerical Tests and Discussion}

In order to compare the BEM and the DRBEM for solving the Helmholtz equation in terms of their numerical accuracy and efficiency, their range applicability in the frequency domain and their reliability in terms of avoiding the well-known "irregular frequencies", two numerical tests were conducted. In the following discussion, for a clear presentation, we shall name

(1) Approach 1: the BEM which takes a Neumann function as the fundamental solution;

(2) Approach 2: the BEM which takes a Hankel function as the fundamental solution;

(3) Approach 3: the DRBEM.

3.1. A unit circle In this test, the boundary geometry is taken to be a unit circle, on which the essential boundary condition $\phi=1$, at $r=1$ is imposed. Obviously, the exact solution for Equation (1.1) subject to this boundary condition is

$$
\phi=\frac{J_{0}(k r)}{J_{0}(k)},
$$

which can be used ideally to check our numerical results. The normal derivative of $\phi$ on the boundary turns out to be a constant,

$$
q=\frac{\partial \phi}{\partial n}=-k \frac{J_{1}(k)}{J_{0}(k)}
$$

where $J_{v}$ denotes the Bessel function of the first kind of $\nu$ th order. 
It can be seen, from the above analytical solution, that at the Dirichlet eigenvalues [9] (which are the natural frequencies of the system), that is, the zeros of $J_{0}$, both $\phi$ and $q$ are infinitely large. A good algorithm should be able to render accurate numerical solutions not only when $k$ is far away from the eigenvalues of the problem but also when $k$ is close to these eigenvalues. Of course, all numerical algorithms should fail at the natural frequencies of the system. The closeness of the correct prediction of a particular algorithm is no doubt an important indicator of the algorithm. We shall certainly examine the behaviours of the BEM and the DRBEM near the eigenvalues of the system.

The boundary was evenly discretized into 32 constant elements for the BEM solution and 24 constant elements for the DRBEM solution, respectively. An additional $24 * 7+1$ internal points used in the DRBEM were placed on eight circles with radii $0.875,0.75,0.625,0.5,0.375,0.25,0.125$ and 0 respectively to guarantee good accuracy of the DRBEM [14]. Although the number of collocation points used in the DRBEM was much larger than that used in the BEM, the CPU times used for a fixed wavenumber $k$ only increased slightly. It was found that the CPU time used in the DRBEM is independent of the value of $k$ (about 2 minutes) (here and hereafter, CPU times all refer to a Sun 4/470 Sparc Server), while the CPU time used in the BEM is dependent on the value of $k$ (approximately 1 minute for Approaches 1 and 2 when $k$ is increased from 0.1 to 8). Furthermore and most importantly, it was found that the CPU time used for calculating 40 values of $k$ (from 0.2 to 8) with Approaches 1-3 being adopted are 35,40 and 13 minutes respectively. Obviously, the DRBEM is much more efficient for eigenvalue analyses.

To measure the numerical errors associated with each of the approaches, only the errors of the calculated $\phi$ and $q$ values on the boundary need to be found [6], since all the values of $\phi$ at any internal point are dependent solely on the full information on the boundary (that is, $\phi$ and $q$ ). In this particular example, only $q$ is unknown on the boundary; therefore we take the maximum error associated with the calculated $q$ values as a measurement and examine its variation with the wavenumber $k$.

The percentage errors of the calculated $q$ terms versus the wavenumber $k$, with Approaches 1 and 3 being adopted respectively, are plotted in Figure 1, while the percentage errors with Approach 2 being adopted are plotted in Figure 2. From these two figures, one can clearly see that both the BEM and the DRBEM can give very accurate results before the first eigenvalue is reached except that there is an irregular frequency associated with Approach 1, at which a large error is shown. Beyond the first eigenvalue, the BEM can still give reasonably accurate results within a large band of spectra, whereas the numerical errors associated with the DRBEM are just too large for the method to be reliable in this frequency range. In fact, if we define $\sigma$ to be the ratio of the sum of the ranges of $k$ values for which the relative errors exceed $10 \%$ to the total range of $k$ values under examination, the $\sigma$ value for Approach 3, with $k$ 


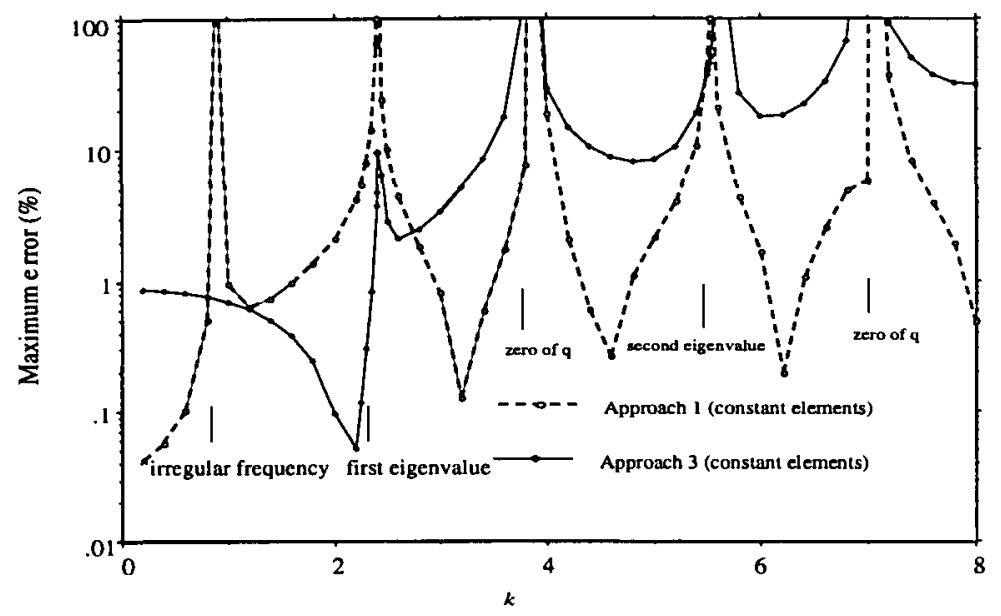

FIGURE 1. Maximum percentage errors of Approaches 1 and 3 versus $k$ in the first numerical test.

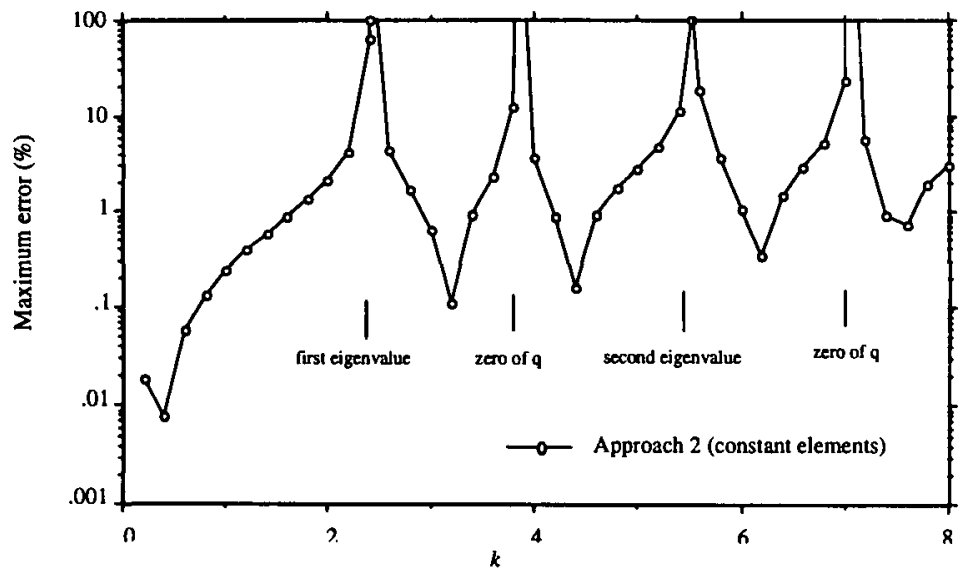

FIGURE 2. Maximum percentage errors of Approach 2 versus $k$ in the first numerical test.

varying between the second eigenvalue and the largest $k$ value so far computed (that is, $k=8$ ), is 1 , whereas the $\sigma$ values for Approaches 1 and 2 in the same range are 0.22 and 0.2 respectively. This simply shows that the DRBEM becomes unreliable beyond the first eigenfrequency and Approach 2 performs the best within this frequency range. One should also notice that large relative errors are shown near the zeros of $q$ (that is, zeros of $J_{1}$ ), which is expected since the values of the exact solutions are very small near these zeros. In fact, the absolute errors near these zeros are not too large (about 0.01).

As far as predicting eigenvalues is concerned, the numerical values obtained from 
the DRBEM seem to have approached infinity at almost the same rate as the exact solution. In fact, near the first eigenvalue $(k=2.40482)$, for the resolution we adopted for the wavenumber $k$, the maximum error detected for the DRBEM solution was just around $10 \%$ ! In order to closely examine the behaviours of these numerical solutions near the eigenvalues of the system, the calculated $\phi$ values near the first and second eigenvalues $(k=2.40482$ and $k=5.5200)$ are tabulated respectively in Tables 1 and 2, from which one can clearly see that all of these approaches can accurately predict the first eigenvalue, because the numerical solutions jump from a very small negative number to a very large positive number as $k$ varies from 2.40482 to 2.41482 (the analytical solution shows that there should be a discontinuous jump of values of $\phi$ from $-\infty$ to $\infty$ across the first eigenvalue $k=2.40482$ ). As for the prediction of the second eigenvalue, Table 2 suggests that Approach 3 can yield a more accurate second eigenvalue than Approaches 1 and 2 can. Our results once again confirmed what has been shown by Partridge and Brebbia [15], that is, as far as the prediction of eigenvalues are concerned, the DRBEM does seem to be much more efficient than the traditional BEM.

TABLE 1. Numerical solutions near the first eigenvalue $(k=2.40482)$.

\begin{tabular}{|c|c|c|c|}
\hline $\mathrm{k}$ & Approach 1 & Approach 2 & Approach 3 \\
\hline 2.39482 & -127 & -127 & -232 \\
\hline 2.40482 & -272 & -271 & -412217 \\
\hline 2.41482 & 2130 & 1726 & 218 \\
\hline
\end{tabular}

TABLE 2. Numerical solutions near the second eigenvalue $(k=5.52007)$.

\begin{tabular}{|c|c|c|c|}
\hline $\mathrm{k}$ & Approach 1 & Approach 2 & Approach 3 \\
\hline 5.51007 & -229 & -221 & -326 \\
\hline 5.52007 & -393 & -319 & -370728 \\
\hline 5.53007 & -1366 & -377 & 268 \\
\hline
\end{tabular}

In addition to rendering larger relative errors near the natural eigenfrequencies and the zeros of $q$ as discussed before, Approach 1 yielded quite large errors near the wavenumber $k=0.89$ too as can be seen from Figure 1 and Table 3 in which the numerical and exact values of $q$ near $k=0.89$ from Approaches 1 and 2 as well as their relative errors are tabulated. Hence $k=0.89$ can only be classified as the wavenumber corresponding to the so-called "irregular frequencies", at or near which all classical integral equation formulations (the direct BEM is one) fail to have a unique solution or have no solution at all due to the presence of large numerical errors which arise, unlike the natural eigenfrequencies associated with a physical phenomenon as discussed in the previous paragraph, entirely as a direct consequence of attempting 
TABLE 3. Numerical solutions near the irregular frequency $(k=0.89)$ associated with Approach 1 in the first numerical test.

\begin{tabular}{|c|c|c|c|c|c|}
\hline $\mathrm{k}$ & Approach 1 & Approach 2 & Exact & Error 1 (\%) & Error 2 (\%) \\
\hline 0.8 & -0.3490 & -0.3468 & -0.3473 & 0.5 & 0.13 \\
\hline 0.897 & -2.1450 & -0.4465 & -0.4473 & 379 & 0.17 \\
\hline 1.0 & -0.5676 & -0.5717 & -0.5730 & 0.95 & 0.23 \\
\hline
\end{tabular}

an integral formulation of the problem. Although these irregular frequencies were often observed when solving exterior Helmholtz problems [2], we can use, following the above definition, the same terminology here for interior problems as well in order to emphasize the fact that similar phenomena exist for interior problems if the fundamental solution is not carefully chosen. Indeed, the large numerical errors which occurred near the irregular frequency in Approach 1 are inherent from the integral equation formulation using the Neumann function as the fundamental solution and have nothing to do with the original problem, which was confirmed by the fact that simply replacing the Neumann function with the Hankel function had eliminated the large errors associated with the irregular frequencies as can be seen in Figure 2. Furthermore, in the literature there are papers in which "irregular frequencies" for interior problems are mentioned and discussed. When looking for the eigenvalues of interior Helmholtz problems using an indirect BIE formulation, it was De Mey [11] who found that to obtain correct eigenvalues, both the real part and imaginary parts of the Hankel function had to be taken into consideration. In a paper on finding eigenvalues of interior Helmholtz problems using a theorem in [10], Adeyeye $e t$ al. [1] also pointed out that if only the imaginary part of the Hankel function, that is, the Neumann function, is taken, "spurious eigenvalues", which we term "irregular frequencies", may occur.

As for how to get rid of these irregular frequencies in a BIE formulation, many "remedies" have been proposed in the past (for example, the Burton and Miller formulation [4] and the method of Schenck [18]). Since a detailed review for most of the newly developed integral formulations designed to avoid "irregular frequencies" for the entire frequency spectrum or only for a range of frequencies can be found in [2], "irregular-frequency-free" BIE formulations shall not be discussed in this paper.

Since the only difference between Approaches 1 and 2 is the different choice of the fundamental solutions to the Helmholtz equation (Equations (2.2) and (2.3)), we therefore conjectured that the adoption of the Neumann function alone as the fundamental solution in Approach 1 and its oscillating behaviour must have resulted in the large numerical errors near certain wavenumbers. In Approach 2, although Bessel functions were again adopted as the fundamental solutions, the complex combination of the two Bessel functions may have some cancellation effects; irregular frequencies 
were not present for the range of frequencies so far examined. To show our conjecture, we plotted out the values of the determinant of matrix $A$ corresponding to the two different fundamental solutions (Equations (2.2) and (2.3)) in Figures 3 and 4 respectively. ( $A$ is the final coefficient matrix of Equation (2.4) after boundary conditions are imposed so that Equation (2.4) can be rewritten in the form $A \boldsymbol{x}=\boldsymbol{b}$ and the determinant of matrix $\boldsymbol{A}$ will be $\operatorname{denoted}$ by $\operatorname{det}(\boldsymbol{A})$ hereafter.) Since a large number of constant boundary elements results in small entries of $A$ so that the round-off error becomes too large for the calculation of $\operatorname{det}(A)$ and also since the main characters of $\operatorname{det}(\boldsymbol{A})$ versus $k$ have little dependence on the number of constant elements adopted in general, the data in Figures 3 and 4 were generated using only 8 constant elements. It is clearly shown in these figures that there exists a zero of $\operatorname{det}(A)$ near $k=0.89$ since $\operatorname{det}(\boldsymbol{A})$ changes its sign when $k$ passes through this point, if $\boldsymbol{A}$ is constructed from the Neumann function (see Figure 3 ). On the other hand, $\operatorname{det}(\boldsymbol{A})$ remains positive if $\boldsymbol{A}$ is constructed from the Hankel function (Figure 4). Furthermore, the wavenumber $k \approx 0.89$ doesn't seem to have affected the accuracy of the DRBEM (see Figure 1), which may be attributed to the monotonicity of the logarithmic function (the weighting function) adopted in the DRBEM. The "irregular-frequency-free" character of the DRBEM approach could also be attributed to the independence of the matrices $\boldsymbol{H}^{D}$ and $G^{D}$ from the wavenumber $k$.

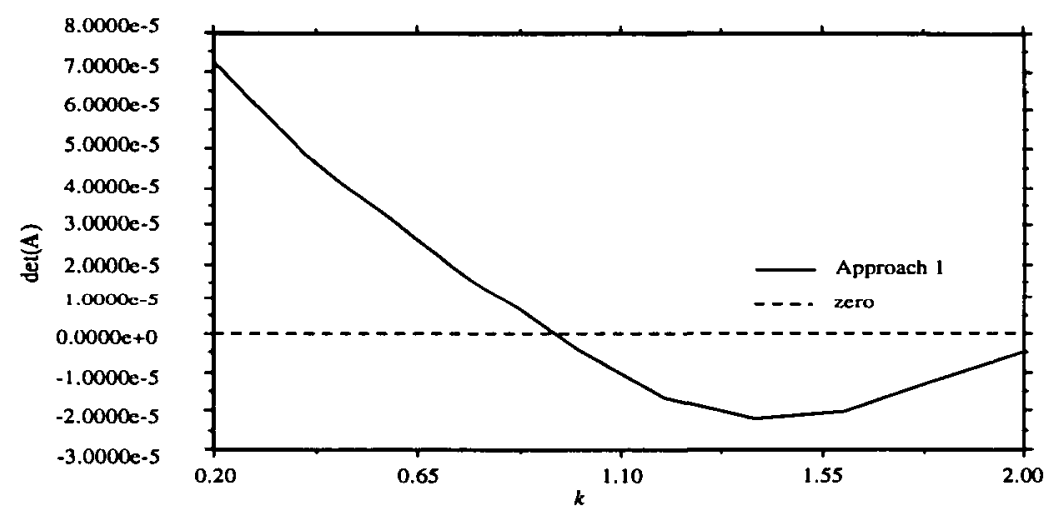

FIGURE 3. $\operatorname{Det}(\boldsymbol{A})$ versus $k$ for Approach 1 in the first numerical test.

Although Approach 2 doesn't seem to have problems associated with irregular frequencies, at least within the range of wavenumbers so far examined, it should be noted that the adoption of the Hankel function as the fundamental solution is computationally more expensive due to the complex matrices and vectors involved in a calculation. As noted, the CPU time used for computing 40 values of $k$ is about 35 minutes for Approach 1 and 40 minutes for Approach 2.

The effects of the number of elements and the type of elements used were also 


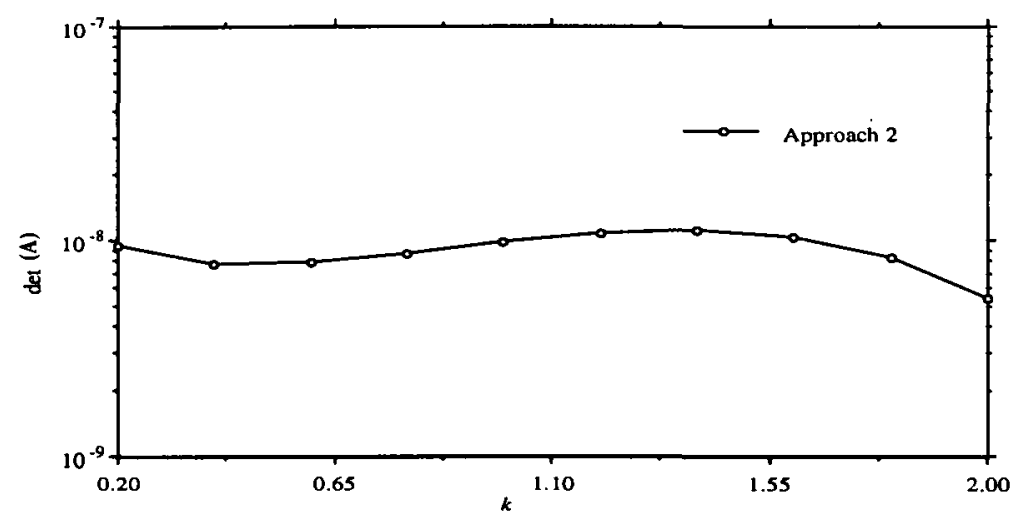

FiguRE 4. $\operatorname{Det}(\boldsymbol{A})$ versus $k$ for Approach 2 in the first numerical test.

examined. It was found that a further increase of boundary elements in the BEM led to improved numerical results (see Figures 5 and 6), whereas a further increase of boundary elements and internal points in the DRBEM resulted in very little further improvement in terms of numerical accuracy. In BEM formulation, the numerical accuracy would be even better if more refined integration schemes are used such as the adoption of higher-order interpolation functions or spline interpolation. See the quadratic elements to be discussed below and see Rezayat et al. [16] for a detailed discussion of spline interpolation. On the other hand, the reason that a further increase of boundary elements and internal points in the DRBEM hardly resulted in any further improvement in terms of numerical accuracy is not yet fully understood, though it is commonly realized by DRBEM researchers that the numerical accuracy associated with the DRBEM is improved for up to a certain amount of collocation points and then deteriorates afterwards [14].

To quantitatively show this feature of the DRBEM, we examined the quality of approximation given by Equation (2.7) (or (2.8)) at a high frequency $k=2.6$ as a response of the number of boundary elements and internal collocation points in Tables 4 and 5 respectively. In these tables, the maximum norm between a function $f$ and its approximate function $\tilde{f}$, which is defined as $\max _{\Omega}|f-\tilde{f}|$, with $\Omega$ being the domain within which both functions are defined, is used as a measurement of the quality of approximation. Similar behaviour was observed when the number of the boundary elements and that of the internal collocation points were simultaneously increased. These tables clearly show that the number of collocation points we used before ( 24 boundary elements and 169 internal collocation points) is actually the "optimal" one; the solution presented in Figure 1 is the best we obtained after a number of numerical tests (including changing the pattern of internal points) had been performed. Therefore we conclude that the DRBEM fails to generate accurate results in a high frequency range (specifically, when $k$ is larger than the first eigenvalue). 


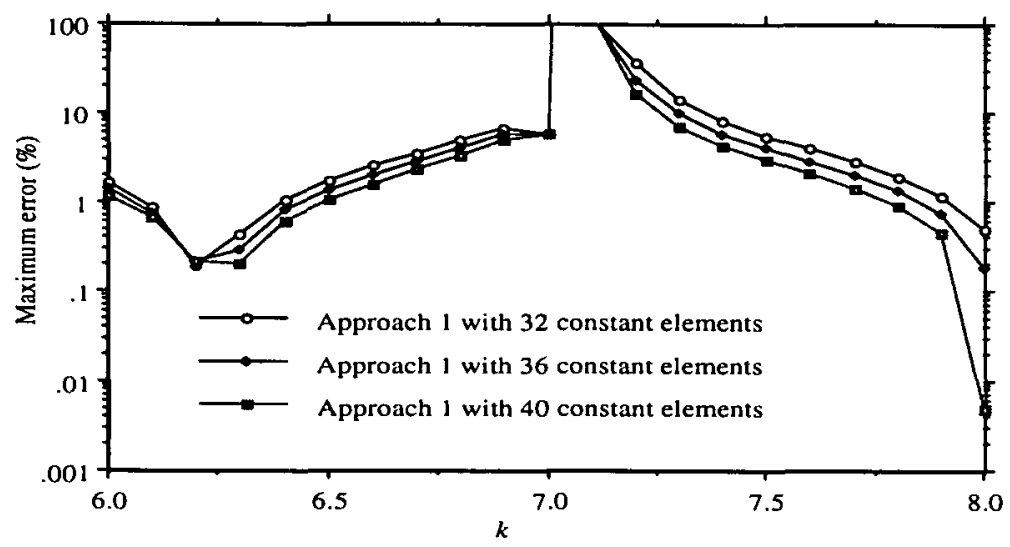

FIGURE 5. Maximum percentage errors of Approach 1 versus the number of boundary elements for high frequency $k$.

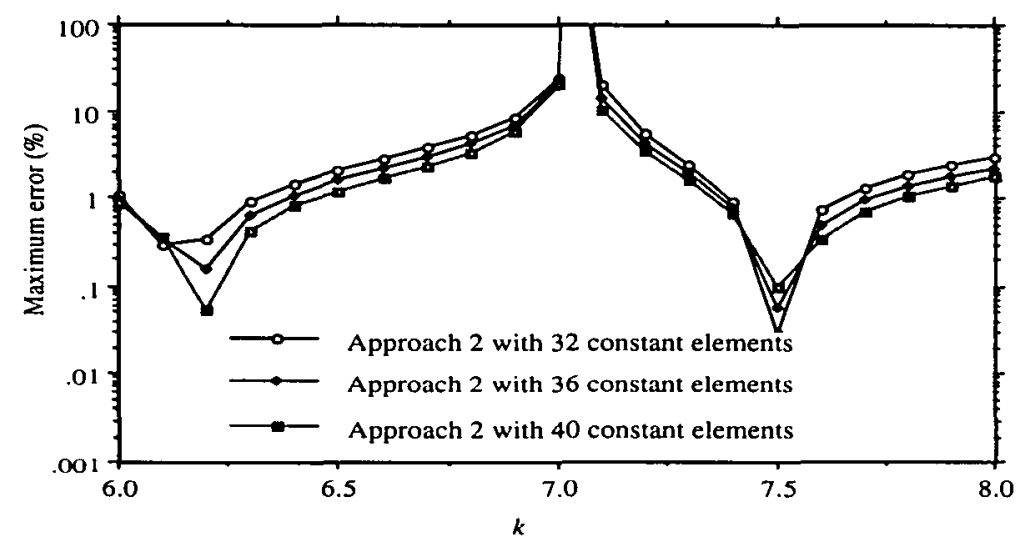

FIGURE 6. Maximum percentage errors of Approach 2 versus the number of boundary elements for high frequency $k$.

TABLE 4. Maximum norms associated with the DRBEM approximation (Equation (2.9)) versus the number of boundary elements ( 169 internal collocation points, $k=2.6$ ).

\begin{tabular}{|c|c|c|c|c|c|c|c|}
\hline Number of b.e. & 24 & 25 & 26 & 27 & 28 & 32 & 40 \\
\hline Maximum norms & 0.0757 & 0.0798 & 0.0799 & 0.0801 & 0.0802 & 0.0805 & 0.0809 \\
\hline
\end{tabular}

TABLE 5. Maximum norms associated with the DRBEM approximation (Equation (2.9)) versus the number of internal collocation points ( 24 boundary elements, $k=2.6$ ).

\begin{tabular}{|c|c|c|c|c|c|}
\hline Number of i.p. & 169 & 170 & 171 & 172 & 174 \\
\hline Maximum norms & 0.0757 & 0.0786 & 0.0784 & 0.0785 & 0.116 \\
\hline
\end{tabular}


To examine the effects of the type of boundary elements used, constant elements were replaced by quadratic elements. Since the boundary integral formulation of Approach 1 has presented one "irregular frequency" even before the first eigenfrequency is reached, further examination of Approach 1 becomes insignificant; computations were only carried out for Approaches 2 and 3 with quadratic elements being adopted. For Approach 2, 16 quadratic elements were placed on the boundary, and for Approach 3,12 quadratic elements were placed on the boundary and the same pattern of internal points as before was chosen inside of the domain. Notice that for these discretizations, the total number of collocation points hasn't been changed. For the DRBEM, the CPU time used for a calculation for a fixed $k$ was about 2.5 minutes, which is a little longer than that with constant elements. For the BEM, the CPU time varies from 1.5 to 2 minutes when $k$ varies from 0.1 to 8 . Again, although CPU times spent for computing results for a particular value of $k$ are close to each other with these two approaches being adopted, CPU times used for computing results for 40 values of $k$ are quite different. Approach 3 is computationally much more efficient than Approach 2 in the latter case.

Similarly, the relative errors associated with calculated $q$ values are plotted out against $k$ in Figure 7. Comparing with Figures 1 and 2, we can see that the adoption of quadratic elements has improved numerical accuracy. Such an improvement is especially evident for the BEM. For the DRBEM, the solution still exhibits large errors at some values of $k$ less than the second eigenvalue (for example, at $k=4$, the error is $11 \%$ ), and at all values of $k$ greater than the second eigenvalue. As a good indicator to show the accuracy of a certain approach within a range $0.2 \leq k \leq 8$, the $\sigma$ values (which have been defined before) for Approaches 2 and 3, with constant elements and quadratic elements being adopted respectively, are tabulated in Table 6, from which we can not only see the improvement by replacing constant elements with quadratic elements but also see that for Approach 3, more than 10\% of relative errors are still present in a large portion of $k$ values even though quadratic elements were adopted. Therefore, if accurate results are required, we recommend that the quadratic elements BEM be adopted, especially when $k$ exceeds the first eigenvalue. We can also conclude now that the DRBEM is only reliable before the first eigenvalue as far as calculating the unknown function values are concerned though it has been shown that accurate eigenvalues can be obtained via the DRBEM.

Such a failure of the DRBEM as the wavenumber $k$ becomes large is a direct consequence of the reformulation of the Helmholtz equation before the DRBEM is applied. In DRBEM formulation, the second term in Equation (1.1) was moved to the righthand side and then approximated by the expansion given in Equation (2.7). However, for the current problem, one can use the analytical solution to show that the solution becomes very oscillatory as $k$ becomes large. Therefore a large number of boundary elements and internal collocation points are needed to resolve the rapid variation of 


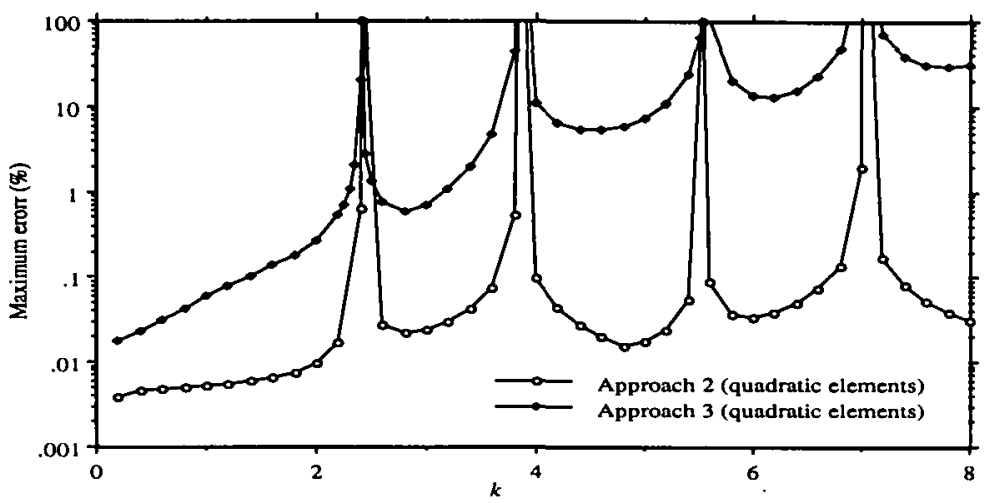

FIGURE 7. Maximum percentage errors of Approaches 2 and 3 using quadratic elements versus $k$ in the first numerical test.

TABLE 6. Values of $\sigma$ for Approaches 2-3 for different types of elements in the first numerical test.

\begin{tabular}{|c|c|c|}
\hline$\sigma$ & constant elements & quadratic elements \\
\hline Approach 2 & 0.15 & 0.047 \\
\hline Approach 3 & 0.48 & 0.42 \\
\hline
\end{tabular}

the solution so that a good approximation could be achieved via Equation (2.7). On the other hand, the numerical manipulation of matrices of very large dimensions inevitably leads to intolerable round-off errors, which impedes the adoption of a large number of boundary elements and internal collocation points. Therefore, the DRBEM with the operator split is restricted to small $k$. In another word, when $k$ is large, the operator $\left(\nabla^{2}+k^{2}\right)$ should be dealt with as a whole.

3.2. A rectangle of slenderness 2 In order to confirm the conclusions we drew in the previous subsection on the DRBEM, we chose our second example to be the same as that used by Partridge and Brebbia [15], who only discussed the calculation of the natural frequencies using the DRBEM. The motion of a forced vibrating beam of rectangular shape with length $a(=2)$ and width $b(=1)$ is governed by Equation (1.1) subject to the following mixed boundary conditions:

$$
\begin{array}{ll}
\phi=0, & \text { for } x=0, \text { and } 0 \leq y \leq 1 ; \\
q=0, & \text { for } y=0, \text { and } 0 \leq x \leq 2 ; \\
q=0, & \text { for } x=2, \text { and } 0 \leq y \leq 1 ; \\
q=1, & \text { for } y=1, \text { and } 0 \leq x \leq 2 .
\end{array}
$$

The exact solution to the differential system (1.1) and (3.1) can be easily obtained by the separation of variables and the natural frequencies for the vibrating beam system 
are simply

$$
\frac{1}{a}\left(n-\frac{1}{2}\right) \pi, \quad n=1,2,3, \ldots .
$$

To find a numerical solution of the problem, the boundary of the rectangle was discretized into boundary elements. To avoid the difficulty of representing normals at the corners, only constant elements were employed. With the BEM, the boundary of the rectangle was discretized into 60 constant elements. With the DRBEM, the same discretization as for the BEM was used on the boundary and in addition, 55 internal points were placed inside of the domain. A schematic view of the DRBEM discretization is shown in Figure 8.

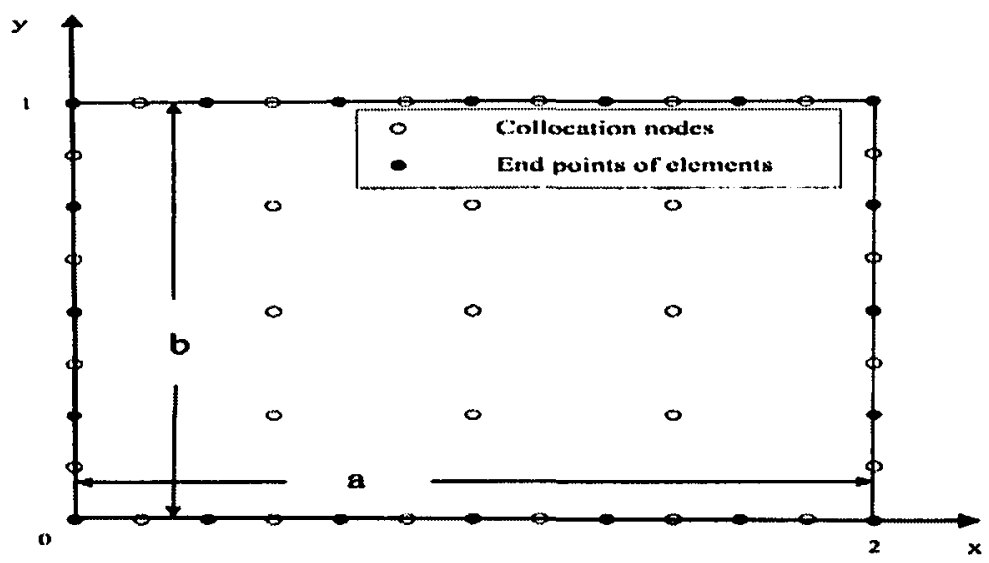

FIGURE 8. A schematic view of the DRBEM discretization used in the second numerical test.

Similar to what has been found in the previous subsection, for a particular value of $k$, it was found that the CPU time is independent of $k$ if the DRBEM is adopted ( 0.5 minutes), and varies from 4 minutes to 6.5 minutes when $k$ is increased from 0.1 to 2, if the BEM (either Approach 1 or 2 ) is adopted. Once again, due to the weighting function adopted in the DRBEM being independent of $k$, the DRBEM seems to be numerically more efficient than the BEM.

To compare the numerical accuracy of the three approaches, we defined a ratio

$$
v=\frac{\text { Number of boundary nodes at which numerical error exceeds } 15 \%}{\text { Total number of boundary nodes }}
$$

and plotted $v$ against $k$ in Figure 9, for Approaches 1 and 3, and in Figure 10, for Approach 2. It is seen that before the first eigenvalue is reached, all three approaches work well, and all of them can correctly predict the first eigenvalue (0.7853). Approach 3 (the DRBEM) breaks down soon after the first eigenvalue. 


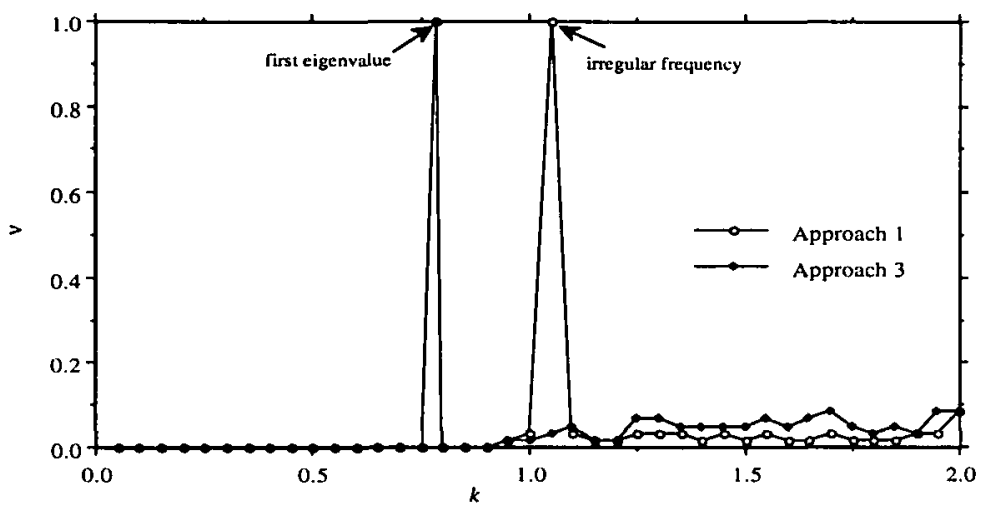

FIGURE 9. The values of $\nu$ for Approaches 1 and 3 versus $k$ in the second numerical test.

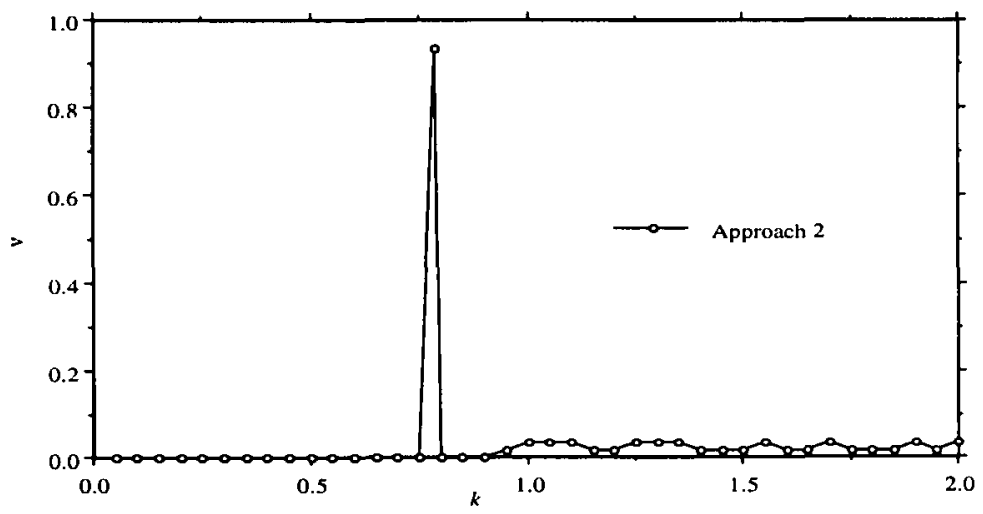

FIGURE 10. The values of $v$ for Approach 2 versus $k$ in the second numerical test.

As shown in Figure 9, there also exists an irregular frequency $k \approx 1.05$, at which Approach 1 breaks down while Approach 2 still works. This is confirmed after the values of $\operatorname{det}(A)$, resulting from Approaches 1 and 2, are plotted against $k$ in Figures 11 and 12 , respectively. (For the same reason given in the previous subsection, only 12 constant elements were used to calculate $\operatorname{det}(\boldsymbol{A})$ shown in Figures 11 and 12.) In addition, the "averaged (over the number of boundary nodes) relative errors" near the irregular frequency with Approaches 1 and 2 being adopted respectively have been tabulated in Table 7, from which one can more clearly see the wrong behaviour of Approach 1 around the irregular frequency. It was also noticed that the DRBEM solution was not affected by the irregular frequency, confirming the conclusion drawn in the previous subsection. Furthermore, eigenfrequencies for higher modes can also be correctly predicted by the DRBEM, as has been demonstrated by Partridge and Brebbia [15], although the DRBEM can no longer yield reliable numerical results as far as solving the unknown function $\phi$ and its normal derivative $q$ is concerned. 


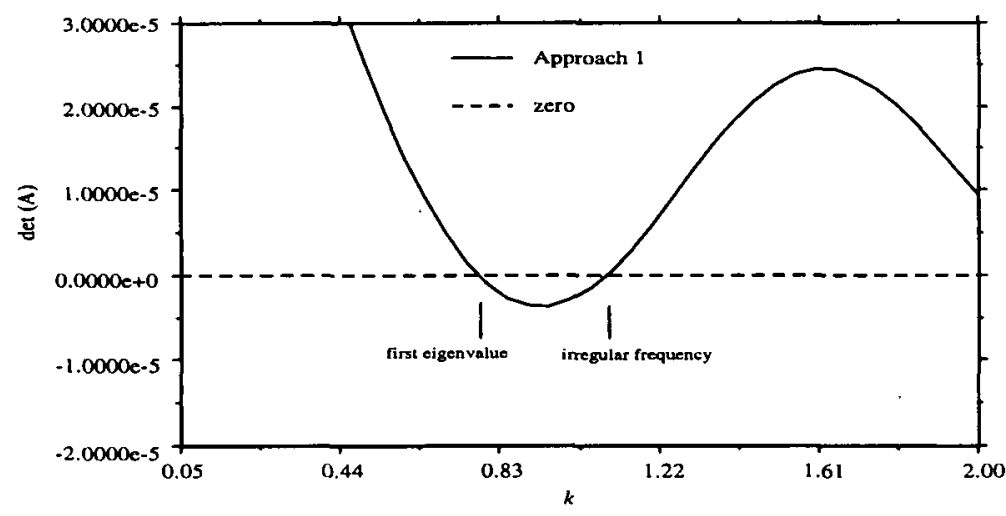

FIGURE 11. $\operatorname{Det}(A)$ versus $k$ for Approach 1 in the second numerical test.

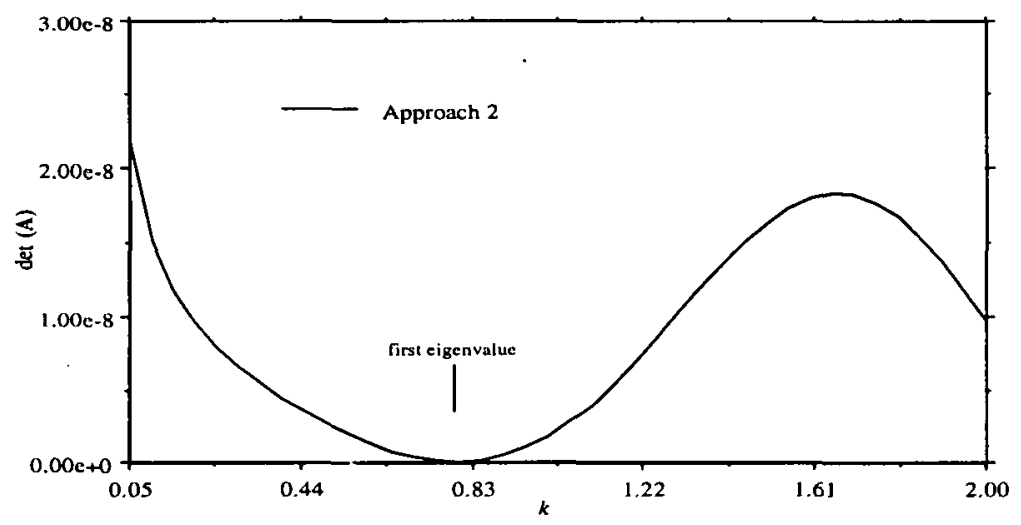

FIGURE 12. $\operatorname{Det}(\boldsymbol{A})$ versus $k$ for Approach 2 in the second numerical test.

TABLE 7. Averaged errors of Approaches 1-2 near the irregular frequency $(k=1.05)$ associated with Approach 1 in the second numerical test.

\begin{tabular}{|c|c|c|}
\hline $\mathrm{k}$ & Approach 1 & Approach 2 \\
\hline 1.00 & $5.9 \%$ & $1.5 \%$ \\
\hline 1.05 & $457 \%$ & $2.1 \%$ \\
\hline 1.10 & $13.6 \%$ & $11.5 \%$ \\
\hline
\end{tabular}

\section{Conclusions}

A comparison of the BEM and the DRBEM in solving the Helmholtz equation has been made in this paper; their accuracy and efficiency, their range of applicability in the frequency domain and their reliability in terms of avoiding the well-known 
"irregular frequencies" have been investigated. Since there are two possible choices of fundamental solutions for a direct BEM formulation of interior Helmholtz problems, that is, a Hankel function or a Neumann function (the real part of a Hankel function) as the weighting function [12], a comparison of the numerical results obtained from these two different fundamental solutions has also been presented in this paper.

It is found that both the BEM and the DRBEM can give very accurate results before the first eigenvalue is reached except that there may exist irregular frequencies associated with the BEM using the Neumann function as the weighting function. Beyond the first eigenvalue, the BEM can still give reasonably accurate results with a large band of frequency spectra, whereas the numerical errors associated with the DRBEM become intolerable.

Computing Bessel functions is often time consuming. Furthermore, since the fundamental solutions used in the BEM involve the wavenumber $k$ which needs to be changed from time to time when the eigenvalues of an interior Helmholtz problem are sought, the numerical efficiency of the BEM in eigenvalue analyses deteriorates. On the other hand, the DRBEM seems to be sufficiently accurate and much more efficient in calculating the eigenvalues. However, if a direct problem is to be solved, the difference between these methods is hardly noticeable in terms of numerical efficiency.

"Irregular frequencies" can be associated with interior problems as well. For both boundary geometries used in this paper, irregular frequencies were found when the BEM using a Neumann function as the fundamental solution was adopted; this approach is therefore obviously not a good choice for solving direct Helmholtz problems. On the other hand, the BEM using a Hankel function as the fundamental solution works well for the whole frequency range explored so far, especially when quadratic elements are used. It is therefore recommended that the BEM using a Hankel function as the fundamental solution be adopted for the numerical calculation of interior Helmholtz problems when the wavenumber is larger than the first eigenvalue. For smaller wavenumbers, the DRBEM is preferred due to its sufficient accuracy and reliability.

\section{References}

[1] J. O. Adeyeye, M. J. M. Bernal and K. E. Pitman, "An improved boundary integral equation method for Helmholtz problems", Int. J. Num. Meth. Engng. 21 (1985) 779-787.

[2] S. Amini, P. J. Harris and D. T. Wilton, Coupled Boundary and Finite Elements Methods for the Solution of the Dynamic Fluid-Structure Interaction Problem, Volume 77 of Lecture Notes in Engineering (Springer-Verlag, Berlin, Heidelberg, New York, 1992).

[3] C. A. Brebbia, J. C. F. Telles and L. C. Wrobel, Boundary Element Techniques (Springer-Verlag, Berlin, 1984). 
[4] A. J. Burton and G. F. Miller, "The application for integral equation methods to the numerical solution of some exterior boundary-value problems", Proc. Roy. Soc. London Ser. A. 323 (1971) 201-210.

[5] D. Colton., "The inverse scattering problem for time-harmonic acoustic waves", SIAM Rev. 26 (1984) 323-350.

[6] M. S. Ingber and A. K. Mitra, "Grid redistribution based on measurable error indicators for direct boundary element method", Engng. Ana. Boundary Elements 9 (1991) 13-19.

[7] N. Kamiya and E. Andoh., "Boundary element eigenvalue analysis by standard routine", Boundary Elements XV 1 (1993) 375-383.

[8] O. D. Kellog, Foundations of Potential Theory (Springer-Verlag, Berlin, 1929).

[9] S. M. Kirkup and S. Amini, "Solution of the Helmholtz eigenvalue problem via the boundary element method", Int. J. Numer. Methods Eng. 36 (1993) 321-330.

[10] R. E. Klainman and G. F. Roach, "Boundary integral equations for three dimensional Helmholtz equation", SIAM Rev. 16 (1974) 214-236.

[11] G. De Mey, "Calculation of eigenvalues of the Helmholtz equation by an integral equation", Int. $J$. Num. Meth. Engng. 10 (1976) 59-66.

[12] G. De Mey., "A simplified integral equation method for the calculation of the eigenvalues of Helmholtz equation", Int. J. Num. Meth. Engng. 10 (1976) 1340-1342.

[13] A. J. Nowak and C. A. Brebbia, "Solving Helmholtz equation by multiple reciprocity method", in Computer and Experiments in Fluid Flow (eds. G. M. Carlomangno and C. A. Brebbia), (Computational Mechanics Publication, Southampton, 1989) 265-270.

[14] A. J. Nowak and P. W. Partridge, "Comparison of the dual reciprocity and the multiple reciprocity methods", Engng. Ana. Boundary Elements 10 (1992) 155-160.

[15] P. W. Partridge and C. A. Brebbia, "The dual reciprocity boundary element method for the Helmholtz equation", in Mechanical and Electrical Engineering (eds. C. A. Brebbia and A. Chaudouet-Miranda), (Computational Mechanics Publication, Southampton, Boston, 1990).

[16] M. Rezayat, D. J. Shippy and F. J. Rizzo, "On time-harmonic elastic wave analysis by the boundary element method for moderate to high frequencies", Comp. Meth. Appl. Mech. Eng. 55 (1986) 349_ 367.

[17] M. G. Salvadori and M. L. Baron, Numerical Methods in Engineering (Prentice-Hall, Englewood Cliffs, N.J., 1961).

[18] H. A. Schenck, "Improved integral formulation for acoustic radiation problems", J. Acoust. Soc. Am. 44 (1968) 41-58.

[19] R. P. Shaw, "Boundary integral equation method applied to wave problems", in Developments in Boundary Element Methods I (eds. P. K. Banerjee and R. Buterfield), (Applied Science Publisher Ltd., London, 1979) Ch. 6, 121-154.

[20] G. R. C. Tai and R. P. Shaw, "Helmholtz equation eigenvalues and eigenmodes for arbitrary domains", J. Acoust. Soc. Am. 56 (1974) 796-804, (also Rep. No. 90, SUNY, Buffalo),

[21] R. Wait and A. R. Mitchell, Finite element analysis and applications (John Wiley and Sons, Chichester, 1985).

[22] S.-P. Zhu and G. Moule, "Numerical calculation of forces induced by short-crested waves on a vertical cylinder of arbitrary cross-sections", Ocean Eng. 21 (1994) 645-662.

[23] O. C. Zienkiewitz and R. L. Taylor, The finite element method, Vols. I and 2 (McGraw-Hill, London, 1988). 